\title{
MEMBERS OF THE COUNCIL
}

1914

\section{OFFICERS}

E. B. VAN Vleck, President.

L. P. Eisenhart, $\}$ Vice-Presidents.

E. J. WILCZYNskI,

F. N. Cole, Secretary.

J. H. TANNER, Treasurer.

D. E. Ммітн, Librarian.

F. N. Cole,

VIRGIL SNYDER, $\}$ Committee of Publication.

J. W. Youna, $\}$

Ex-Presidents

J. H. Van Amringe, Emory McClintock, G. W. HiLL,
R. S. WOODWARD,

E. H. MOORE,

T. S. FIsKe,

W. F. OsGood,
H. S. White, MAXIME BÔCHER,

H. B. Fine.

\section{Elected Members}

To serve until December, 1914
A. B. Coble,
Oswald Veblen,
E. W. Davis,
E. B. WILson.

To serve until December, 1915
F. C. Ferry,
R. C. Maclaurin,
W. B. FORD, J ACOB WESTLUND.

To serve until December, 1916
C. N. HASKINS,
E. V. Huntington,
L. M. Hoskins,
H. L. RIETz.

Editortal Committee of the Transactions
L. E. Dickson,
D. R. Curtiss,
P. F. SмiтH. 